\title{
Next generation sequencing and molecular imaging identify EGFR mutation and amplification in a glioblastoma multiforme patient treated with an EGFR inhibitor: a case report
}

\author{
Ke Zhou ${ }^{1,3, *}$, Hui Yao ${ }^{1, *}$, Xuewen Zhang ${ }^{1}$, Jiangang Liu ${ }^{1}$, Zhenyu $\mathbf{Q i}^{1}$, Xueshun Xie ${ }^{1}$, \\ Xiaoting $X u^{2}$, Youxin Zhou ${ }^{1}$, Zhengquan $\mathbf{Y u}^{1}{ }^{1}$, Zhong Wang ${ }^{1}$, Yanjun Che ${ }^{3}$ and Yulun \\ Huang $^{1}$ \\ ${ }^{1}$ Department of Neurosurgery and Brain and Nerve Research Laboratory, The First Affiliated Hospital of Soochow University, \\ Suzhou, China \\ 2 Department of Radiotherapy, The First Affiliated Hospital of Soochow University, Suzhou, China \\ ${ }^{3}$ Department of Neurosurgery, The Jingjiang People's Hospital, Taizhou, China \\ * These authors have contributed equally to this work \\ Correspondence to: Yulun Huang, email: huangyulun@suda.edu.cn
}

Yanjun Che, email: cheyj2003@163.com

Keywords: EGFR mutation; glioblastoma; molecular image; erlotinib; next-generation sequencing

Received: January 16, $2017 \quad$ Accepted: April 14, 2017

Published: May 24, 2017

Copyright: Zhou et al. This is an open-access article distributed under the terms of the Creative Commons Attribution License 3.0 (CC BY 3.0), which permits unrestricted use, distribution, and reproduction in any medium, provided the original author and source are credited.

\section{ABSTRACT}

Epidermal growth factor receptor (EGFR) mutations and amplifications are frequently reported in glioblastoma multiforme (GBM) patients. In this case report, we utilize next-generation sequencing (NGS) and EGFR molecular imaging to investigate intratumoral heterogeneity in a male patient presenting with GBM. Further, we describe the patient's clinical course as well as outcomes of targeted EGFR therapy with erlotinib, an EGFR tyrosine kinase inhibitor (TKI). NGS demonstrated the presence of an EGFR mutation and amplification in our patient. Molecular imaging revealed a heterogeneous expression pattern of EGFR in the frontal and temporal lobes. This patient briefly responded to erlotinib therapy. However, the patient relapsed and died from progressive neurological deterioration. Partial response and acquired secondary resistance may be attributed to intratumoral heterogeneity. Combination of NGS and EGFR molecular imaging may be helpful in understanding intratumoral molecular heterogeneity and may aid in developing individualized GBM treatments, thereby improving outcomes.

\section{INTRODUCTION}

Glioblastoma multiforme (GBM) is a highly malignant tumor of the central nervous system. The overall median survival time of GBM is approximately $12-15$ months [1, 2]. Currently, GBM is primarily treated with surgical resection, followed by a combination of radiotherapy and chemotherapy.

Epidermal growth factor receptor (EGFR) is a transmembrane receptor tyrosine kinase protein that has been associated with several human malignancies. EGFR associated mutations, amplification or overexpression are observed in approximately $50 \%$ of glioblastoma patients [3]. EGFR amplification has been proposed as a marker of poor prognosis [4], however, EGFR-targeted therapy can be a promising treatment for GBM patients.

Erlotinib (Tarceva) is an oral EGFR tyrosine kinase inhibitor (TKI) that has been extensively clinically validated. However, patients' response rate ranges from 10 to $20 \%$ [5-7]. Phase II clinical trials revealed that the single-agent activity of erlotinib was marginally beneficial for GBM patients following radiotherapy [5]. Moreover, erlotinib demonstrated insufficient singleagent activity over standard therapies in GBM patients [8]. Therefore, identifying markers that can predict the outcome of erlotinib therapy may be beneficial for GBM patients and might uncover factors that improve treatment sensitivity. PTEN is a tumor suppressor gene that is 
commonly mutated in glioblastoma [9]. The expression of amplified and aberrant EGFR combined with the expression of wildtype PTEN were important predictors for the sensitivity towards EGFR kinase inhibition in glioblastoma xenografts [10]. In GBM patients, coexpression of EGFRvIII and PTEN was significantly associated with a favorable clinical response [11]. Moreover, GBM patients with higher levels of EGFR expression and lower levels of phosphorylated PKB/Akt demonstrated improved sensitivity to erlotinib treatment [12]. Nevertheless, to date, the molecular characteristics of GBM subpopulations of patients that demonstrate higher responses towards TKIs have not been fully elucidated [13]. Here we describe a GBM patient who had a shortterm response to TKI. We used next generation sequencing (NGS) and molecular imaging to investigate the presence of EGFR mutations.

\section{CASE PRESENTATION}

A 31-year-old male was admitted to the First Affiliated Hospital of Soochow University, China complaining from headache, vomiting, and mild left hemiparesis. Magnetic resonance imaging (MRI) revealed a large abnormal mass in the left temporal parietal area with marked edema and a shift of the midline structures to the left side (Figure 1A and 1B). The patient was diagnosed with glioblastoma and underwent gross total resection in February 2015. The tumor tissue was preserved for immunohistochemical study which revealed immunopositive reactions against the GBM biomarkers GFAP, CD56, vimentin, nestin and Olig-2 (Figure 2A, $2 \mathrm{~B}$ and $2 \mathrm{C}$ ). Further, the ki67 labeling index was $70 \%$ (Figure 2D). The gross total resection of the GBM was confirmed by a follow up MRI performed at one month post-operatively (Figure 1C and 1D). The patient received treatment according to Stuup et al [14] regimen of standard radiation and concomitant temozolomide chemotherapy. After radiation, MRI verified that the patient had not relapsed (Figure 1E and 1F). His therapeutic regimen was composed of six adjuvant temozolomide cycles (first cycle was $150 \mathrm{mg} / \mathrm{m}^{2} /$ day, the remainder of the cycles were 200 $\mathrm{mg} / \mathrm{m}^{2} /$ day) for five days every 28 days. By September 2015, our patient successfully finished five cycles of adjuvant temozolomide, however, routine follow up MRI revealed the relapse of the GBM prior to commencement of the sixth temozolomide cycle (Figure $1 \mathrm{G}$ and $1 \mathrm{H}$ ).

Subsequently, we performed NGS by using tissues obtained at diagnosis in order to investigate the molecular characteristics of the temozolomide resistant GBM and to identity new therapeutic strategies. Total DNA was extracted from tumor paraffin sections using the GeneRead ${ }^{\mathrm{TM}}$ DNA FFPE Kit (Qiagen, Germany), according to the manufacturer's protocol. Genomic DNA was fragmented into fragments ranging from 300-350 bp using a focused-ultrasonoscope (Covaris M220, USA).
Agilent SureSelect XT reagents were used to prepare sequencing libraries according to the manufacturer's protocol. Hybrid capture was conducted using Agilent SureSelectXT Human All Exon V6. After PCR amplification, the library was created using Bioanalyzer 2100 (Agilent, USA) and AriaMx Real-Time PCR system (Agilent, USA). The library was sequenced on Illumina HiSeq4000 Analyzers (Illumina, USA) for 151 cycles to generate $150 \mathrm{bp}$ paired-end reads. Image analysis and base calling were performed using the Illumina Pipeline. Sequencing depth was $500-8000 X$. NGS revealed the following results: EFGR amplification 14 times, EGFR p.A289V mutation, pP772 delinsPP mutation and FLNA point mutation (Table 1). Further, data analysis identified EGFR as a driver gene.

Next, we evaluated EGFR expression in the patient's brain by molecular imaging at eight months post-operatively. EGFR antibody-18-FDG was used as a tracer agent. We injected the EGFR antibody-18-FDG and performed a PET/CT scan at 24, 48, 72 hours postinjection. We observed significant tracer uptake by the tumor at 24, 48, and 72 hours. The standardized uptake value (SUVmax) of tumor/ non-tumor was 6.4, 10, and 8.2 for the 24, 48 and 72 hours, respectively (Figure 3 and data not shown). Furthermore, the temporal lobe of the brain showed a strong positive signal while the frontal lobe showed a weak positive signal.

Given the above-mentioned results, we decided to administer TRI therapy with erlotinib $150 \mathrm{mg} /$ day starting from November 2015. The patient showed improvement over four days; he showed no dysarthria and no gait abnormalities. MRI scan revealed tumor hollowing and improvement in edema at 14 days posterlotinib treatment (Figure 4A and 4B). Follow up MRI scans were carried out monthly. In the first three months, GBM tumor was improved with less observed edema and reduced compression of the lateral ventricles (Figure 4C and 4D). However, at the fourth month, our patient should progressive neurological deterioration and the MRI scan revealed the recurrence of GBM. In the frontal lobe, the tumor progressed rapidly, extended to corpus callosum and to the right lobe (Figure 4E and 4F). The patient died after three months from the secondary progression in May 2016. Overall survival time was 15 months for the patient. Progression of the patient's illness and the therapeutic regimen are summarized in Table 2

The patient provided informed written consent for the publication of this case report and all accompanying images.

\section{DISCUSSION}

GMB is often associated with the mutation and amplification of the EGFR. Further, EGFR p.A289V mutation is most common mutation associated with GBM [15]. However, the presence of multiple or heterogeneous 

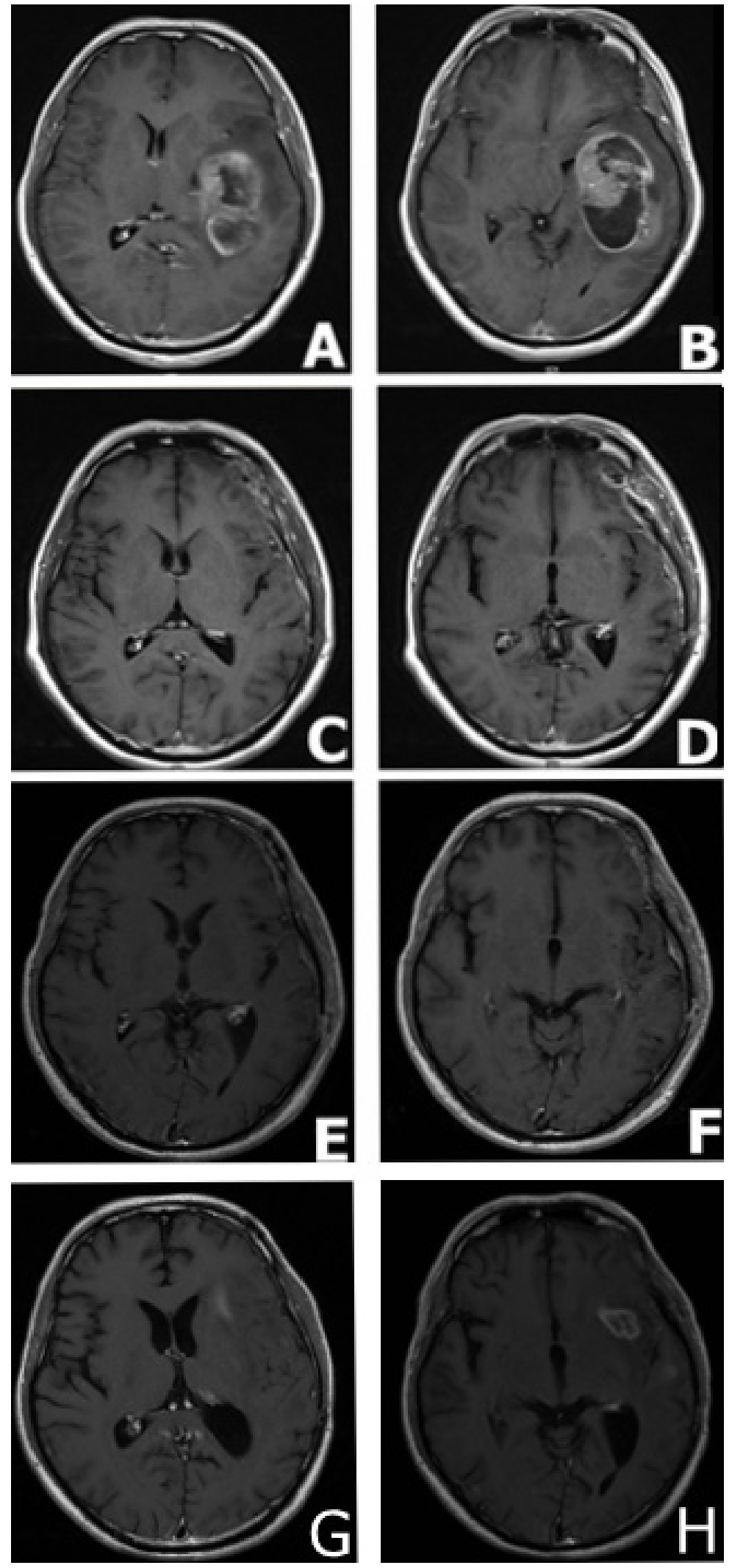

Figure 1: MRI findings in a male patient presented with glioblastoma multiforme. A. and B. MRI scans at the disease onset demonstrating a large mass in the left temporal parietal area with marked surrounding edema and a shift of the midline structures to the left side. C. and D. MRI scans captured at one month following surgical resection; MRI demonstrating gross total resection. E. and $\mathbf{F}$. MRI scans at 5 months following surgical resection, standard radiation and concomitant chemotherapy demonstrated the absence of tumor relapse. G. and H. MRI scans at 7 months following surgical resection: MRI demonstrated tumor relapse. 
Table 1: Mutation analysis of glioblastoma multiforme patient tumor

\begin{tabular}{|l|l|l|l|l|l|}
\hline Gene & Variation type & Nucleotide variation & $\begin{array}{l}\text { Amino acid } \\
\text { variations }\end{array}$ & $\begin{array}{l}\text { Sequencing } \\
\text { depth }\end{array}$ & $\begin{array}{l}\text { Mutation } \\
\text { frequency }\end{array}$ \\
\hline EGFR & point mutation & c.C866T & p.A289V & 7941 & $95 \%$ \\
\hline EGFR & insertion mutation & c.2318_2319insACC & p.P773delinsPP & 7932 & $9.50 \%$ \\
\hline FLNA & point mutation & c.G3718A & p.V1240M & 250 & $20.80 \%$ \\
\hline
\end{tabular}

EGFR gene amplification 14 times

Table 2: Progression of the patient's illness and the therapeutic regimen

\begin{tabular}{|l|l|}
\hline Time & Patient history and treatment regimen \\
\hline Feb, 2015 & $\begin{array}{l}\text { Headache, vomiting, and mild left hemiparesis. The patient was diagnosed with glioblastoma } \\
\text { and underwent gross total resection. }\end{array}$ \\
\hline March/April, 2015 & Standard radiation and concomitant temozolomide chemotherapy. \\
\hline May, 2015-Sept, 2015 & Adjuvant temozolomide 5 cycles (150mg/day in first cycle, 200mg/day in other cycle) \\
\hline Sept, 2015 & The relapse of the GBM \\
\hline Oct, 2015 & Next generation sequencing demonstrates EGFR mutation \\
\hline Oct, 2015 & Perform a PET/CT by EGFR antibody-18-FDG shows non-uniform EGFR positive reaction \\
\hline Nov, 2015 & Oral administration of erlotinib 150 mg/day \\
\hline Nov, 2015-Feb, 2016 & SD (less edema and reduced compression of the lateral ventricles ) \\
\hline March, 2016 & Recurrence of GBM \\
\hline May,2016 & Patient death \\
\hline
\end{tabular}

GBM: Glioblastoma multiforme SD: Stable Disease

types of mutations is a hallmark of GBM. Using deep sequencing, Kumar et al. previously identified spatial heterogeneity in Tp53,EGFR, and PDGFRA genes in glial tumors [16]. Furthermore, Francis et al., developed a novel approach to identify distinct tumor subpopulations from the bulk tumor using single-cell whole-genome sequencing allowing them to infer the subclonal architecture. Detailed mutational analysis of tumors will aide in identifying mechanisms underpinning drug resistance which may ultimately improve the effectiveness of personalized cancer treatment [17]. The overexpression of EGFR is a predictive biomarker for response to different therapeutic regimens. Indeed, molecular imaging is a noninvasive technique that enables the detection of EGFR overexpression. Therefore, molecular imaging can aid in identifying the patient population with positive EGFR expression and hence can benefit from targeted TKI-based therapies.

In this case report, we described the molecular imaging and NGS in a male patient presenting with GBM. Results of EGFR-18F-FDG PET/CT scan demonstrated heterogeneous expression of EFGR protein in our male patient. We observed heterogeneity in the intensity of
EGFR staining across the brain. The temporal lobe of the brain showed a strong positive signal while the frontal lobe showed a weakly positive signal. It is plausible to speculate that the heterogeneity of EFGR expression may result in variable efficacy of TKI therapy [18]. Further, NGS revealed the presence of EFGR amplification and the EGFR mutations p.A289V and pP772 delinsPP1, as well as the FLNA point mutation p.V120M. In the first three months of oral erlotinib treatment, the patient's symptoms improved. However, the efficacy of single erlotinib therapy declined with a relapse in the patient's condition. In the frontal lobe, the GBM expanded, extending to the corpus callosum and to the contralateral lobe. As discussed earlier, the frontal lobe showed lower EGFR activity which may have affected sensitivity towards erlotinib, leading to secondary resistance.

The existence of intratumoral heterogeneity has been demonstrated to possess prognostic implications and may affect the sensitivity towards chemotherapy [19]. Further, intratumorral diversity represented a probable cause for anti-EGFR therapeutic secondary resistance. Due to trimming of tissues obtained from surgical resection, tumor samples do not reflect the molecular 
representation of the whole tumor [19]. Moreover, Wei et al. previously demonstrated that tumors evolve in response to targeted therapies through the classical Darwinian selection and cellular adaptations at a variety of levels [18]. In this study, we demonstrated that the combination of NGS and molecule imaging may be instrumental in studying the complex molecular biology and intratumoral molecular heterogeneity. Further, we observed that single target inactivation was not sufficient to block downstream oncogenic signaling. Our male patient responded briefly to erlotinib before acquiring secondary resistance. It has been previously demonstrated that combining erlotinib
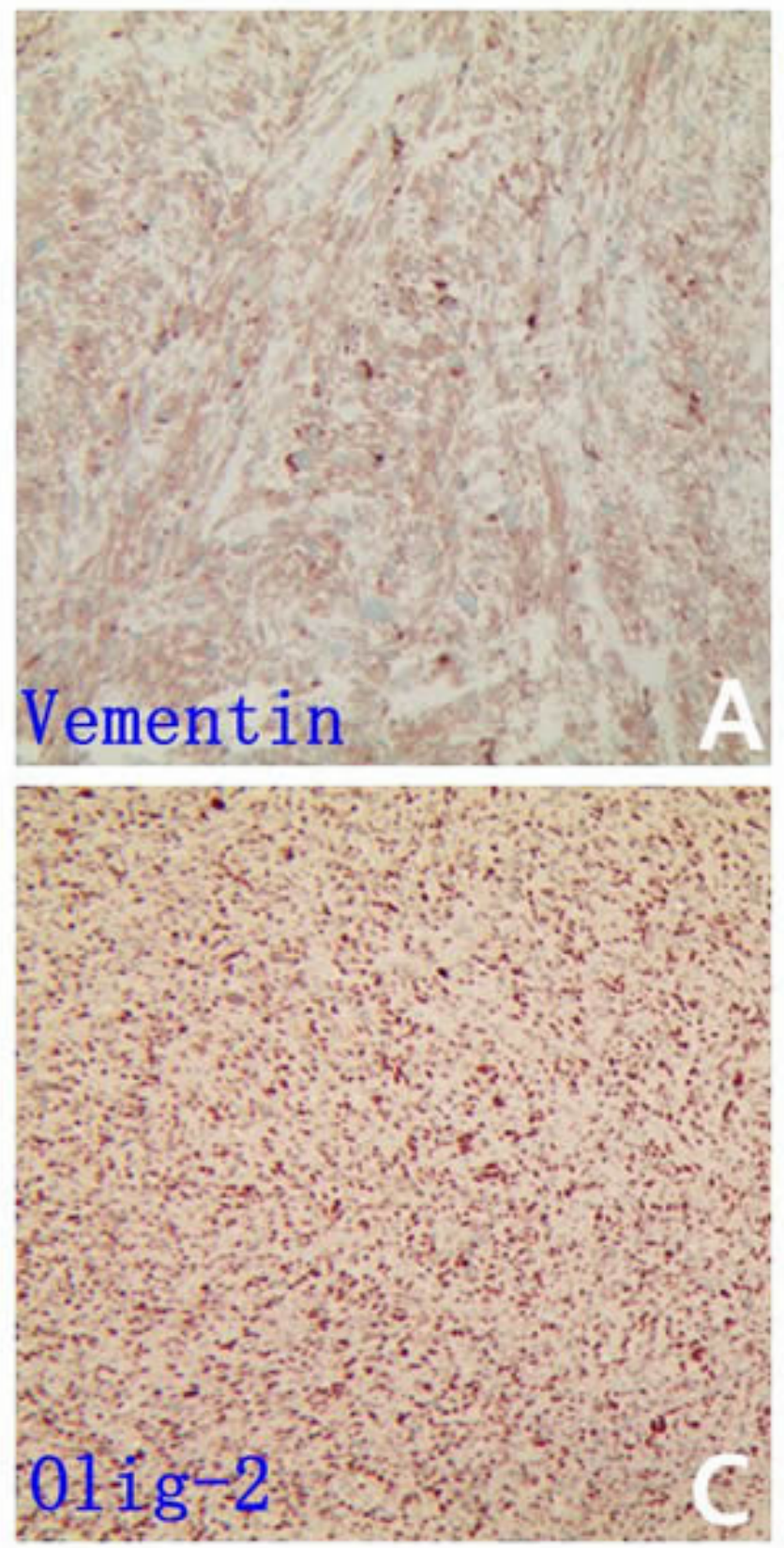

with histone deacetylase inhibitors (HDACi), significantly inhibit the proliferation of erlotinib-resistant GBM cells and partially restore their sensitivity to erlotinib [20]. Moreover, targeting MET, a hepatocyte growth factor receptor, in GBM cases with EGFR amplification may delay the acquired secondary resistance that can develop during erlotinib treatment [21]. Taken together, the combination of erlotinib with other drugs can potentially prevent TKI secondary resistance. Therefore, we recommend combining two or more target drugs which may aide in prolonging survival in GBM patients [18].

In conclusion, NGS and molecular imaging
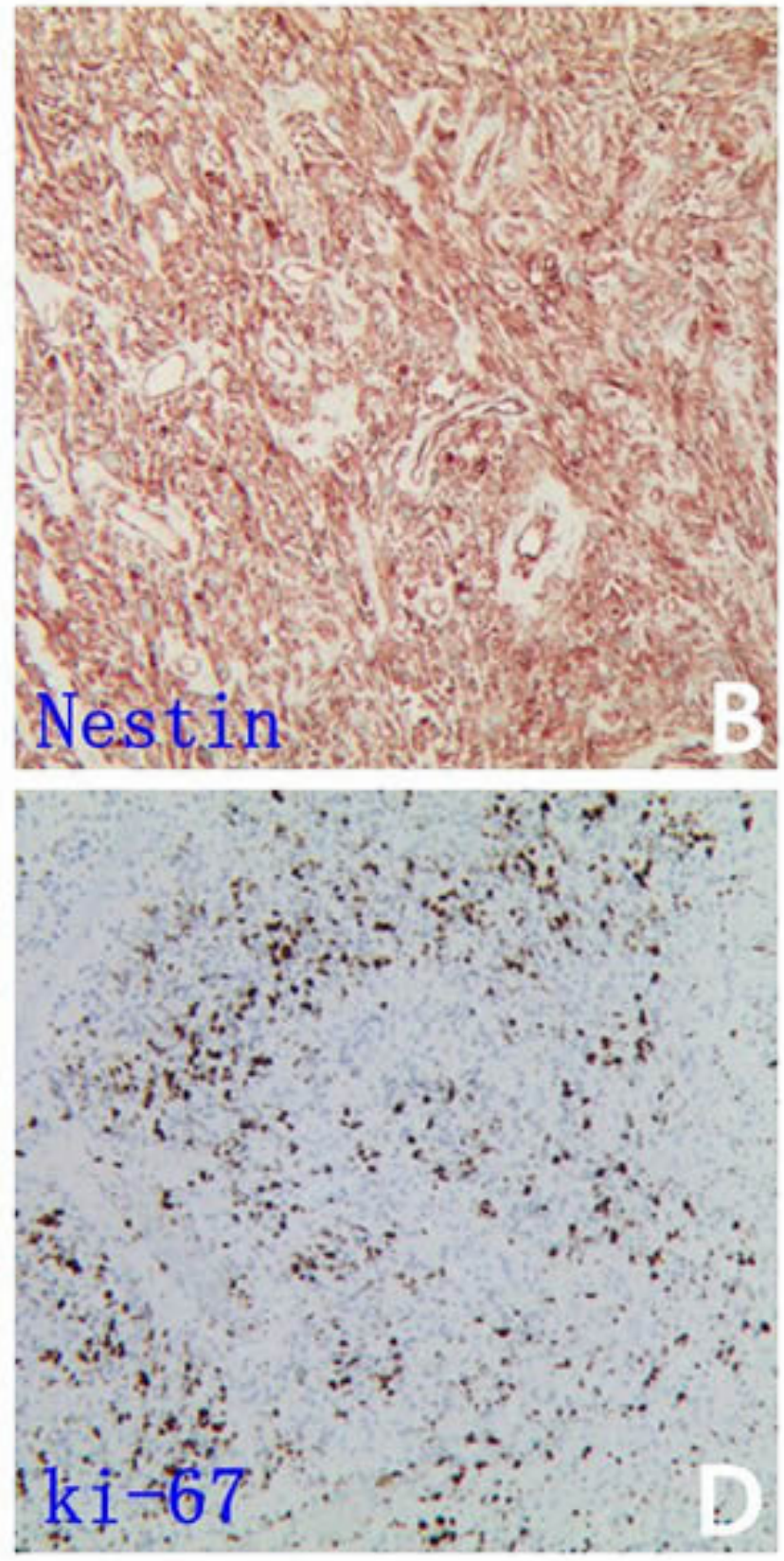

Firgure 2: GBM biomarkers is immunopositive in patient specimen. Representative IHC images are shown for vimentin A., nestin B., Olig-2 C. and the ki67 D. (which labeling index was $70 \%$ ). 

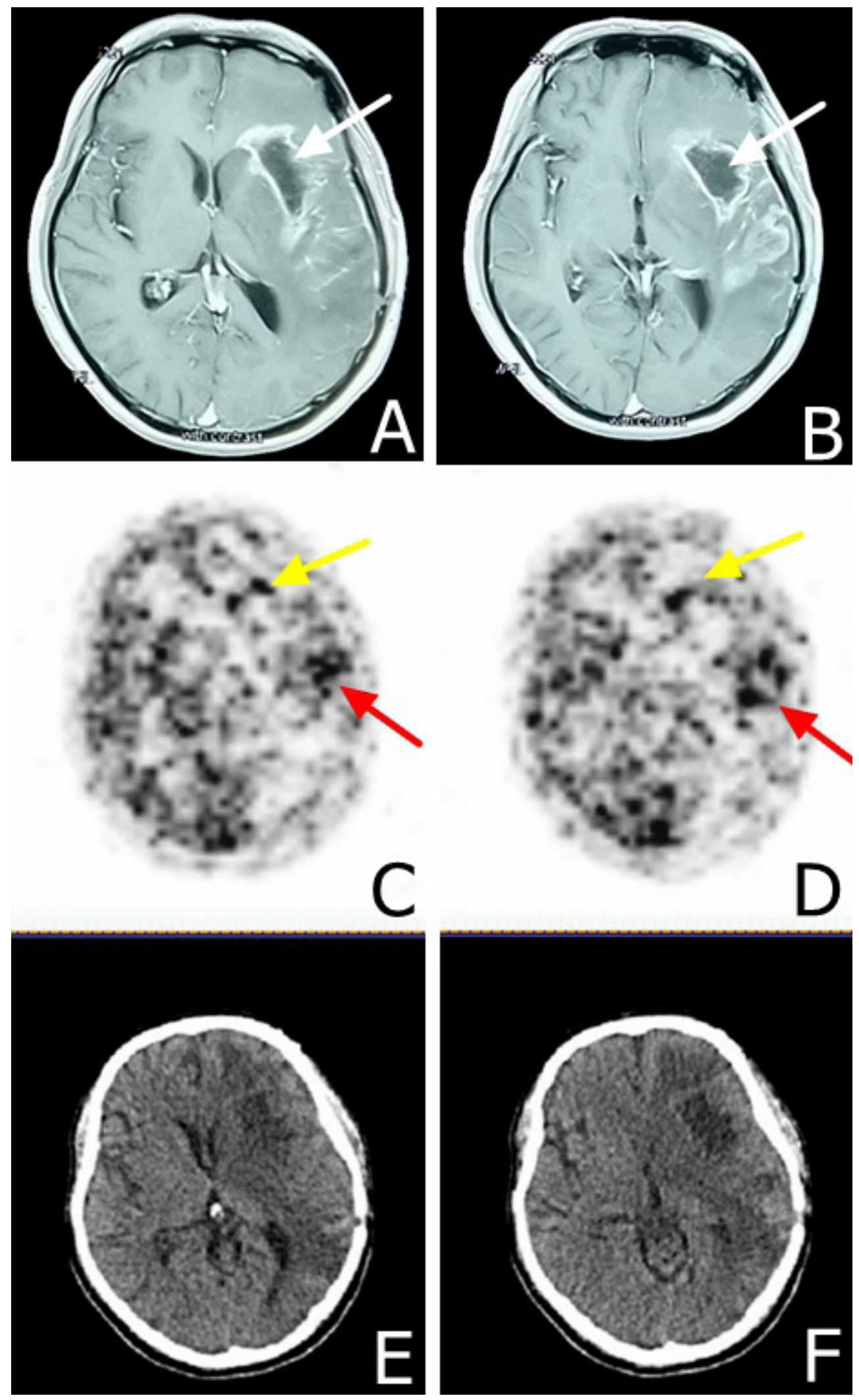

Figure 3: MRI and EGFR-18F-FDG and PET/CT scans in a male patient presented with glioblastoma multiforme at eight months following surgical resection. A. and B. MRI scans demonstrating the tumor relapse at eight months following surgical resection. The white arrow shows the tumor hollowing. C. and D. EGFR-18F-FDG, PET/CT scans demonstrate heterogeneous tumor characteristics at 48 hours post-tracer injection, yellow arrows demonstrate area with lower staining intensity in the frontal lobe and the red arrow demonstrates a higher staining intensity in the temporal lobe. E. and F. CT scan indicating tumor hollowing and significant edema. 

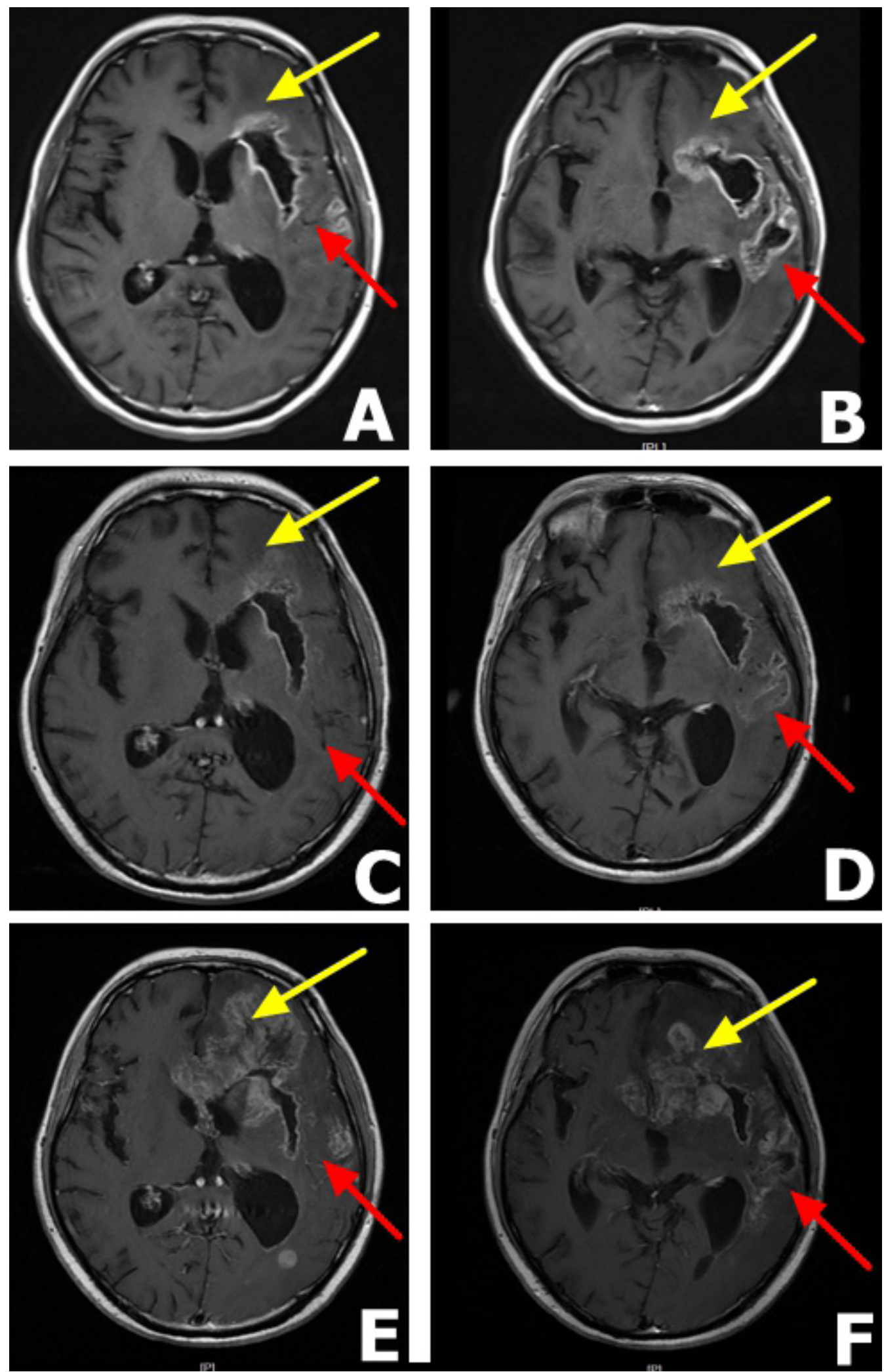

Figure 4: MRI findings in a male patient presenting with glioblastoma multiforme after erlotinib $150 \mathrm{mg}$ therapy A. and B. MRI scans after 14 days of daily erlotinib $150 \mathrm{mg}$ therapy. The scans reveal tumor hollowing, lower grade edema and reduced compression of the lateral ventricles. C. and D. Three months after erlotinib $150 \mathrm{mg}$ therapy, MRI scans shows that the tumor stabilized. Yellow arrow shows a stable frontal lobe while the red arrow show partial response in the temporal lobe. E. and F. Five months after erlotinib $150 \mathrm{mg}$ therapy. MRI scans demonstrate the tumor relapsed for the second time with distant metastasis and the patient died two months later. The yellow arrow shows obvious metastasis in the frontal lobe and red arrow shows a stable temporal lobe. 
can provide crucial information about the molecular composition of tumors which will help in developing individualized targeted therapy.

\section{ACKNOWLEDGMENTS}

This study was supported by the National Natural Science Foundation of China (Grant No. NSFC81372689) , Health and family planning commission of Jiangsu Province youth research subject (Q201606), Six talent peaks project in Jiangsu Province (2014-wsw-021) and Suzhou applied basic research (Sys201535).

\section{CONFLICTS OF INTEREST}

The authors declare that they have no conflict of interests.

\section{REFERENCES}

1. Lim SK, Llaguno SR, McKay RM, Parada LF. Glioblastoma multiforme: a perspective on recent findings in human cancer and mouse models. BMB Rep. 2011; 44:158-164.

2. Ma X, Lv Y, Liu J, Wang D, Huang Q, Wang X, Li G, Xu S, Li X. Survival analysis of 205 patients with glioblastoma multiforme: clinical characteristics, treatment and prognosis in China. J Clin Neurosci. 2009; 16:1595-1598.

3. Frederick L, Wang XY, Eley G, James CD. Diversity and frequency of epidermal growth factor receptor mutations in human glioblastomas. Cancer Res. 2000; 60:1383-1387.

4. Huncharek M, Kupelnick B. Epidermal growth factor receptor gene amplification as a prognostic marker in glioblastoma multiforme: results of a meta-analysis. Oncol Res. 2000; 12:107-112.

5. Raizer JJ, Abrey LE, Lassman AB, Chang SM, Lamborn KR, Kuhn JG, Yung WK, Gilbert MR, Aldape KA, Wen PY, Fine HA, Mehta M, Deangelis LM, et al. A phase II trial of erlotinib in patients with recurrent malignant gliomas and nonprogressive glioblastoma multiforme postradiation therapy. Neuro Oncol. 2010; 12:95-103.

6. Halatsch ME, Gehrke EE, Vougioukas VI, Bötefür IC, A-Borhani F, Efferth T, Gebhart E, Domhof S, Schmidt $\mathrm{U}$, Buchfelder M. Inverse correlation of epidermal growth factor receptor messenger RNA induction and suppression of anchorage-independent growth by OSI-774, an epidermal growth factor receptor tyrosine kinase inhibitor, in glioblastoma multiforme cell lines. J Neurosurg. 2004; 100:523-533.

7. Addeo R, Zappavigna S, Parlato C, Caraglia M. Erlotinib: early clinical development in brain cancer. Expert Opin Investig Drugs. 2014; 23:1027-1037.

8. van den Bent MJ, Brandes AA, Rampling R, Kouwenhoven MC, Kros JM, Carpentier AF, Clement PM, Frenay M,
Campone M, Baurain JF, Armand JP, Taphoorn MJ, Tosoni A, et al. Randomized phase II trial of erlotinib versus temozolomide or carmustine in recurrent glioblastoma: EORTC brain tumor group study 26034. J Clin Oncol. 2009; 27:1268-1274.

9. Cantley LC, Neel BG. New insights into tumor suppression: PTEN suppresses tumor formation by restraining the phosphoinositide 3-kinase/AKT pathway. Proc Natl Acad Sci U S A. 1999; 96:4240-4245.

10. Sarkaria JN, Yang L, Grogan PT, Kitange GJ, Carlson BL, Schroeder MA, Galanis E, Giannini C, Wu W, Dinca $\mathrm{EB}$, James CD. Identification of molecular characteristics correlated with glioblastoma sensitivity to EGFR kinase inhibition through use of an intracranial xenograft test panel. Mol Cancer Ther. 2007; 6:1167-1174.

11. Mellinghoff IK, Wang MY, Vivanco I, Haas-Kogan DA, Zhu S, Dia EQ, Lu KV, Yoshimoto K, Huang JH, Chute DJ, Riggs BL, Horvath S, Liau LM, et al. Molecular determinants of the response of glioblastomas to EGFR kinase inhibitors. N Engl J Med. 2005; 353:2012-2024.

12. Haas-Kogan DA, Prados MD, Tihan T, Eberhard DA, Jelluma N, Arvold ND, Baumber R, Lamborn KR, Kapadia A, Malec M, Berger MS, Stokoe D. Epidermal growth factor receptor, protein kinase $\mathrm{B} / \mathrm{Akt}$, and glioma response to erlotinib. J Natl Cancer Inst. 2005; 97:880-887.

13. Thorne AH, Zanca C, Furnari F. Epidermal growth factor receptor targeting and challenges in glioblastoma. Neuro Oncol. 2016;18:914-918.

14. Stupp R, Mason WP, van den Bent MJ, Weller M, Fisher B, Taphoorn MJ, Belanger K, Brandes AA, Marosi C, Bogdahn U, Curschmann J, Janzer RC, Ludwin SK, et al. Radiotherapy plus concomitant and adjuvant temozolomide for glioblastoma. N Engl J Med. 2005; 352:987-996.

15. Brennan CW, Verhaak RG, McKenna A, Campos B, Noushmehr H, Salama SR, Zheng S, Chakravarty D, Sanborn JZ, Berman SH, Beroukhim R, Bernard B, Wu CJ, et al. The somatic genomic landscape of glioblastoma. Cell. 2013; 155:462-477.

16. Kumar A, Boyle EA, Tokita M, Mikheev AM, Sanger MC, Girard E, Silber JR, Gonzalez-Cuyar LF, Hiatt JB, Adey A, Lee C, Kitzman JO, Born DE, et al. Deep sequencing of multiple regions of glial tumors reveals spatial heterogeneity for mutations in clinically relevant genes. Genome Biol. 2014; 15:530.

17. Francis JM, Zhang CZ, Maire CL, Jung J, Manzo VE, Adalsteinsson VA, Homer H, Haidar S, Blumenstiel B, Pedamallu CS, Ligon AH, Love JC, Meyerson M, et al. EGFR variant heterogeneity in glioblastoma resolved through single-nucleus sequencing. Cancer Discov. 2014; 4:956-971.

18. Wei W, Shin YS, Xue M, Matsutani T, Masui K, Yang H, Ikegami S, Gu Y, Herrmann K, Johnson D, Ding X, Hwang K, Kim J, et al. Single-Cell Phosphoproteomics Resolves Adaptive Signaling Dynamics and Informs Targeted 
Combination Therapy in Glioblastoma. Cancer cell. 2016; 29:563-573.

19. Patel AP, Tirosh I, Trombetta JJ, Shalek AK, Gillespie SM, Wakimoto H, Cahill DP, Nahed BV, Curry WT, Martuza RL, Louis DN, Rozenblatt-Rosen O, Suva ML, et al. Single-cell RNA-seq highlights intratumoral heterogeneity in primary glioblastoma. Science. 2014; 344:1396-1401.

20. Liffers K, Kolbe K, Westphal M, Lamszus K, Schulte A. Histone Deacetylase Inhibitors Resensitize EGFR/ EGFRvIII-Overexpressing, Erlotinib-Resistant Glioblastoma Cells to Tyrosine Kinase Inhibition. Target Oncol. 2016; 11:29-40.
21. Johnson J, Ascierto ML, Mittal S, Newsome D, Kang L, Briggs M, Tanner K, Marincola FM, Berens ME, Vande Woude GF, Xie Q. Genomic profiling of a Hepatocyte growth factor-dependent signature for MET-targeted therapy in glioblastoma. J Transl Med. 2015; 13:306. 